\title{
Relationship Between Brachial Artery Flow-Mediated Dilation, Carotid Artery Intima-Media Thickness and Coronary Flow Reserve in Patients With Coronary Artery Disease
}

\author{
Fahrettin Oz ${ }^{\mathrm{a}, \mathrm{b}}$, Ali Elitok ${ }^{\mathrm{a}}$, Ahmet Kaya Bilge ${ }^{\mathrm{a}}$, Fehmi Mercanoglu ${ }^{\mathrm{a}}$, Huseyin Oflaz ${ }^{\mathrm{a}}$
}

\begin{abstract}
Background: The aim of this study was to assess the relationship between brachial artery flow mediated dilation (FMD), carotid artery intima-media thickness (IMT) and coronary flow reserve (CFR) in patients with coronary artery disease (CAD).

Methods: Fifty patients with coronary artery disease, except left anterior descending artery (LAD), who showed no cardiac symptoms and 45 control subjects underwent assessment of brachial artery FMD, carotid artery intima-media thickness by high-resolution ultrasound. In addition, transthoracic second harmonic Doppler echocardiography was used to measure CFR.
\end{abstract}

Results: All of the parameters were found to be correlated with each other. CFR correlated with brachial artery FMD $(\mathrm{r}=0.232, \mathrm{P}$ $<0.05)$ and with carotid IMT $(\mathrm{r}=-0.403, \mathrm{P}<0.001)$. Carotid IMT correlated with brachial artery FMD $(\mathrm{r}=-0.211, \mathrm{P}<0.05)$.

Conclusions: Transthoracic CFR correlated with well-established noninvasive predictors of atherosclerosis and we suggest that it can be used as a surrogate for coronary atherosclerosis.

Keywords: Endothelial dysfunction; Atherosclerosis; Coronary artery disease; Coronary flow reserve; Flow mediated dilation; $\mathrm{Ca}-$ rotid artery intima-media thickness

\section{Introduction}

Endothelial dysfunction, by impairing vasomotor tonus,

Manuscript accepted for publication September 28, 2012

${ }^{\mathrm{a}}$ Department of Cardiology, Istanbul University, Istanbul Faculty of Medicine, Istanbul, Turkey

${ }^{\mathrm{b}}$ Corresponding author: Fahrettin Oz, Istanbul Universitesi, Istanbul Tip Fakultesi, Kardiyoloji Anabilim Dali, 34093, Capa, Istanbul, Turkey.

Email: fahrettin_oz@hotmail.com

doi: http://dx.doi.org/10.4021/cr219w promoting arterial thrombosis, vascular cell migration and proliferation is a major determinant of atherosclerosis [1,2]. Endothelial dysfunction is an early and reversible key event of cardiovascular disease and has been used to predict future coronary artery disease prior to atherosclerotic changes in arteries has occured [1]. Inflammation has a critical role in the pathogenesis of atherosclerosis and endothelial dysfunction $[1,2]$. Coronary atherosclerosis is often clinically silent, with death as its first manifestation. Much effort has been made to detect the beginning of the disease. Because primary risk reduction is effective, several noninvasive options to assess atherosclerosis in its preclinical stages have been introduced into clinical use. These include carotid artery intima-media thickness (IMT), brachial artery flow-mediated dilation (FMD). Recently transthoracic second harmonic Doppler echocardiography has evolved as a tool to investigate coronary flow pattern and coronary flow reserve (CFR). Endothelial dysfunction precedes clinically manifested atherosclerosis and its assessment through the use of high-resolution ultrasound to measure brachial artery diameter in response to reactive hyperemia has increased the clinical relevance of vascular function evaluation [3]. High-resolution ultrasound provided evidence that reduced endothelium-mediated vasodilation is associated with increased risk for cardiovascular events in a variety of conditions [4]. In patients with coronary artery disease endothelium-mediated vasodilation of the brachial artery correlates with the coronary vasodilator response [5]. Normally coronary blood flow can increase approximately four-to-six fold to meet increasing myocardial oxygen demands. This effect is mediated by vasodilation at the arteriolar bed, which reduces vascular resistance, thereby augmenting flow. The coronary reserve represents the capacity of the coronary circulation to dilate, following an increase in myocardial metabolic demands and can be expressed by the difference between the hyperemic flow and the resting flow [6]. Coronary flow reserve which depends on endothelium mediated vasoreactivity, also may be impaired in the absence of manifested CAD, and the evaluation of CFR may allow early detection of coronary atherosclerosis [6]. Although CFR was used to be measured invasively until recently, CFR has been evaluated in echo-lab by using Doppler and vasodilator stress such as dipyridamole or adenozine [6]. 
Table 1. Clinical Characteristics of the Subjects and CAD Patients

\begin{tabular}{|c|c|c|c|}
\hline & $\operatorname{CAD}(n=50)$ & Control $(n=45)$ & $\mathbf{P}$ \\
\hline Age (year) & $52.4 \pm 6.4$ & $49.8 \pm 8.9$ & NS \\
\hline Male/Female & $40 / 10$ & $36 / 9$ & NS \\
\hline BMI $\left(\mathrm{kg} / \mathrm{m}^{2}\right)$ & $26.2 \pm 3.44$ & $26.1 \pm 2.94$ & NS \\
\hline Hypertension & $32(64 \%)$ & $9(20 \%)$ & $<0.05$ \\
\hline Diabetes Mellitus & $7(14 \%)$ & - & \\
\hline Smoke & $37(74 \%)$ & $30(66.7 \%)$ & NS \\
\hline Systolic pressure (mmHg) & $126.9 \pm 15.76$ & $123 \pm 17.16$ & 0.39 \\
\hline Diastolic pressure (mmHg) & $79.2 \pm 9.64$ & $77.44 \pm 8.38$ & 0.19 \\
\hline Total Chol (mg/dL) & $186.9 \pm 41.5$ & $195.4 \pm 30.8$ & NS \\
\hline Triglyceride (mg/dL) & $158.18 \pm 84$ & $138.08 \pm 38.51$ & NS \\
\hline $\mathrm{HDL}(\mathrm{mg} / \mathrm{dL})$ & $38.24 \pm 8.71$ & $42.75 \pm 6.06$ & $<0.05$ \\
\hline $\mathrm{LDL}(\mathrm{mg} / \mathrm{dL})$ & $112.04 \pm 34.69$ & $130.02 \pm 27.78$ & $<0.05$ \\
\hline Creatinin (mg/dL) & $0.94 \pm 0.23$ & $0.83 \pm 0.13$ & NS \\
\hline $\mathrm{Hgb}(\mathrm{g} / \mathrm{dL})$ & $13.7 \pm 1.03$ & $14.09 \pm 1.61$ & NS \\
\hline \multicolumn{4}{|l|}{ Medical Treatment } \\
\hline ACE inhibitor & $35(70 \%)$ & $5(20 \%)$ & $<0.001$ \\
\hline Beta blocker & $37(74 \%)$ & 3 & $<0.001$ \\
\hline Calcium C. blocker & $2(4 \%)$ & 1 & NS \\
\hline Aspirin & $48(96 \%)$ & 2 & $<0.0001$ \\
\hline Statin & $45(90 \%)$ & 1 & $<0.0001$ \\
\hline
\end{tabular}

NS: not significant; BMI: Body-Mass Index.

By this method, impairment of CFR can be assessed before development of angiographically detectable stenosis in epicardial coronary arteries and we are able to investigate early coronary microvasculature pathology [7]. Measurement of CFR has predictive power for future cardiovascular events and therefore could be used as a surrogate marker for early atherosclerotic changes of coronary arteries $[6,7]$.

\section{Methods}

We studied 50 patients (40 men; mean age $52.4 \pm 6.4$ y) who had evidence of CAD at coronary angiography. Fortytwo patients underwent coronary angiography because of stable angina pectoris and 8 in consequence of prior myocardial infarction (at least 1 year ago and not anterior MI). Forty five subjects (36 men; mean age $49.8 \pm 8.9$ y) who did not have structural heart disease and who were referred for functional evaluation of chest pain represented the control group. Control group subjects were evaluated with treadmill exercise electrocardiogram testing and none of them were excluded owing to ischemic ECG changes in response to exercise.

All CAD patients and control subjects underwent evaluation after withdrawal of all cardiac drugs for $\geq 18 \mathrm{~h}$ before the study. All abstained from smoking and intake of caffeinecontaining food for at least $24 \mathrm{~h}$ before the study. The ethical committee approved the protocol, and each patient or control subject gave informed consent.

\section{FMD evaluation by brachial artery high-resolution ul- trasound imaging}

Flow mediated dilation was measured according to recent guidelines [8] with ultrasound unit electronic calipers (VIV- 
Table 2. Echocardiographical Features of the Control and the CAD Patients

\begin{tabular}{llll}
\hline & CAD $(\mathbf{n}=\mathbf{5 0})$ & Control $(\mathbf{n}=\mathbf{4 5})$ & $\mathbf{P}$ \\
\hline EF $(\%)$ & $56 \% \pm 7.33$ & $64 \% \pm 4.59$ & $<0.05$ \\
LVMI $\left(\mathrm{g} / \mathrm{m}^{2}\right)$ & $109 \pm 25.48$ & $85.74 \pm 16.91$ & $<0.005$ \\
$\operatorname{IMK}(\mathrm{mm})$ & $0.89 \pm 0.24$ & $0.64 \pm 0.17$ & $<0.005$ \\
FMD $(\%)$ & $5.03 \pm 4.24$ & $7.47 \pm 2.94$ & $=0.02$ \\
BPDV $(\mathrm{cm} / \mathrm{sn})$ & $32.68 \pm 10.20$ & $29.37 \pm 8.49$ & $=0.09$ \\
HPDV $(\mathrm{cm} / \mathrm{sn})$ & $55.88 \pm 17.58$ & $69.13 \pm 27.41$ & $=0.06$ \\
CFR & $1.75 \pm 0.46$ & $2.40 \pm 0.80$ & $<0.005$ \\
\hline
\end{tabular}

BPDV: blsal peak diastolic velocity; HPDV: Hyperemic peak diastolic velocity.

ID 7 General Electric, Wisconsin, and USA) and $10 \mathrm{MHz}$ linear array transducer. To best visualize the brachial artery, the arm was comfortably immobilized in the extended position and the brachial artery was scanned in a longitudinal section $3-5 \mathrm{~cm}$ above the antecubital fossa. After optimal transducer positioning, the skin was marked for reference for later measurements. Three consecutive measurements obtained through consecutive cardiac cycles were averaged and recorded. Briefly, FMD was assessed by measuring the change in brachial artery diameter after $60 \mathrm{~s}$ of reactive hyperemia compared with a baseline measurement after deflation of a cuff that had been placed around the forearm and that had been inflated to $50 \mathrm{mmHg}$ above systolic blood pressure for $5 \mathrm{~min}$. The response of the vessel diameter to reactive hyperemia was expressed as the percent change rela- tive to the diameter immediately before cuff inflation. Flow mediated dilation was expressed as the percentages change in the brachial artery internal diameter from baseline following reactive hyperemia.

\section{Measurements of carotid intima-media thickness}

Carotid arteries provide a window to the coronary arteries. Patients with major carotid stenosis are very likely to have major coronary stenosis. Measurements of carotid IMT with ultrasound is a noninvasive and highly reproducible technique for quantifying atherosclerotic burden. That is the relationship between the atherosclerotic burden in a carotid artery and coronary artery is the same as between any two coronary arteries [9]. Considering the high correlation be-

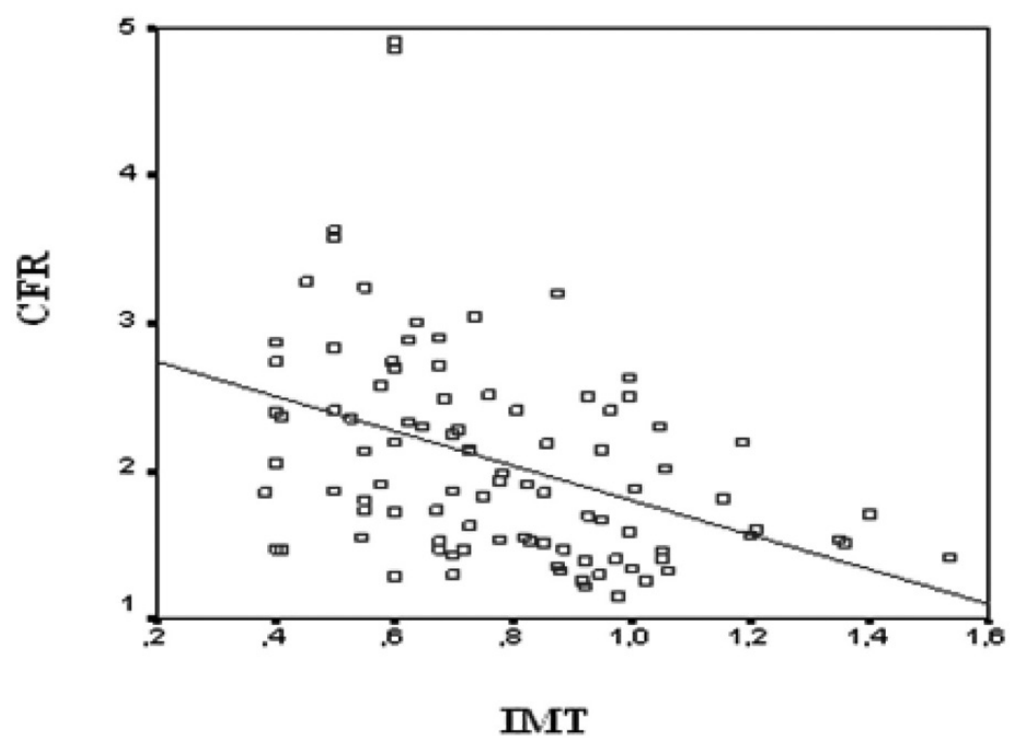

Figure 1. Relationship between coronary flow reserve (CFR) values and intima-media thickness of all subjects in study group $(r=-0.403, P<0.001)$. 


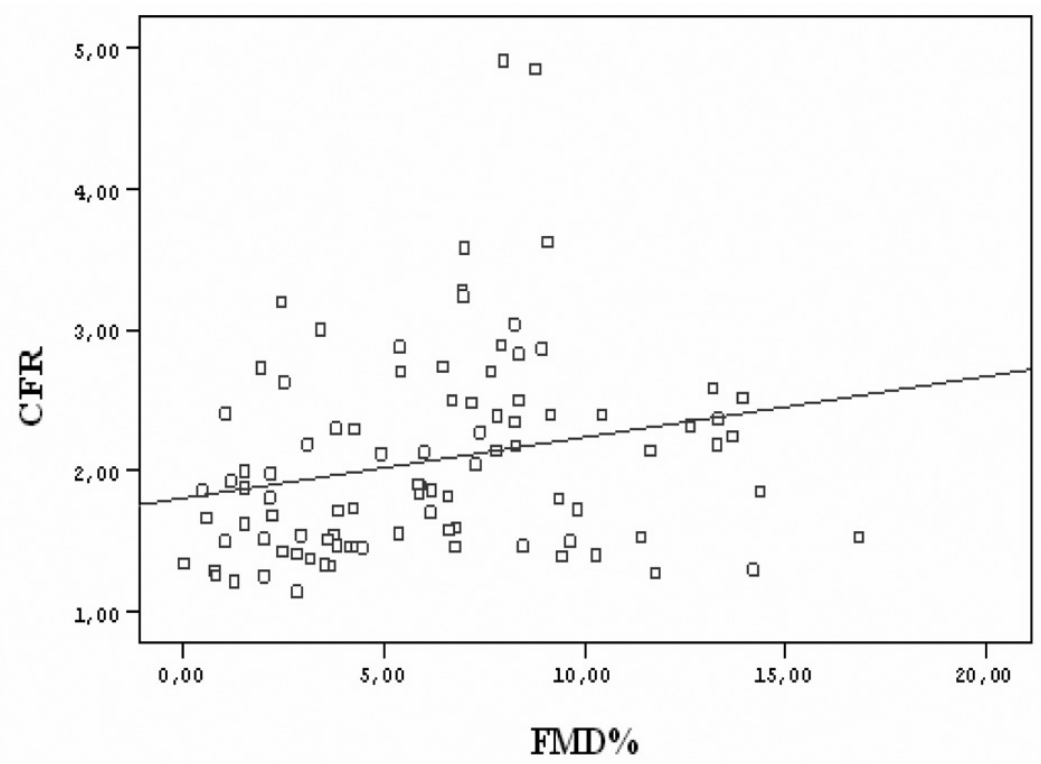

Figure 2. Relationship between coronary flow reserve (CFR) values and flow mediated dilation (FMD) of all subjects in study group $(r=0.232, \mathrm{P}<0.05)$.

tween carotid and coronary artery disease, carotid screening is useful in patients with coronary artery disease. In patients with an occasional finding of a carotid risk score of at least 2 (IMT more than $0.90 \mathrm{~mm}$, unstable plaque and severe stenosis $>70 \%$ ) a careful search for coronary artery disease seems warranted [10]. Carotid IMT has predictive power for future myocardial infarction and stroke beyond the wellknown coronary risk factors [11, 12]. Accordingly American Heart Association (AHA) prevention conference has recommended carotid IMT scanning for patients older than 45 years who require further clarification of their coronary heart disease risk [12]. In this study carotid IMT was measured according to the method described previously $[9,11]$. With the subject in the supine position, longitudinal scanning was performed from the common carotid artery to the bifurcation point. After the bifurcation of the common carotid artery had been confirmed, carotid IMT was measured from the far wall of the internal carotid artery within $10 \mathrm{~mm}$ proximal to the bifurcation. Three points were measured on one scan, which was synchronized with $\mathrm{R}$ wave peaks on the ECG to avoid possible errors resulting from variable arterial compliance. Mean carotid IMT was calculated from six measurements on two scans.

\section{Transthoracic coronary flow measurement}

Coronary flow reserve measurements are used to assess epicardial coronary arteries and to examine the integrity of coronary microvascular circulation. In recent years, transthoracic second harmonic Doppler echocardiographic examination of CFR has become very populer and its feasibility has been validated [13-15]. In this study CFR recordings were per- formed with the Vivid 7 echocardiography device (General Electrics, Wisconsin, and USA) using a middle-range frequency ( $3-8 \mathrm{MHz}$ ) broadband transducer. Visualization of the distal LAD was performed using a modified, foreshortened; two chamber view obtained by sliding the transducer on the upper part and medially, from two-chamber view. Subsequently, coronary flow in the distal LAD was examined by color Doppler flow mapping over the epicardial part of the anterior wall, with the color Doppler velocity range set in the range of $9-24 \mathrm{~cm} / \mathrm{sn}$. The acoustic window was around the midclavicular line, in the fourth and fifth intercostal spaces, with the subject in the left lateral decubitus position $[16,17]$. By placing the sample volume on the color signal, spectral Doppler of the LAD showed the characteristic biphasic flow pattern, with larger diastolic and smaller systolic components. After baseline recordings of flows, dipyridamole ( 0.56 $\mathrm{mg} / \mathrm{kg}$, Persantin, Boehringer Ingelheim, Barcelona, Spain) was infused over a 4-min. period. Two minutes after the end of the infusion, hyperemic spectral profiles in the LAD artery were recorded. By averaging the three highest Doppler signals for each measurement, CFR was defined as the ratio hyperemic to baseline diastolic peak velocities. CFR $>2$ was considered normal $[16,17]$.

\section{Statistical analysis}

Continuous data were expressed as mean $\pm \mathrm{SD}$, and categorical data were expressed as percentages. Chi square-test for categorical variables was used to assess differences among groups. The relationships among parameters were assessed by pearson correlation analysis. A $\mathrm{P}$ value of $<0.05$ was considered statistically significant. 


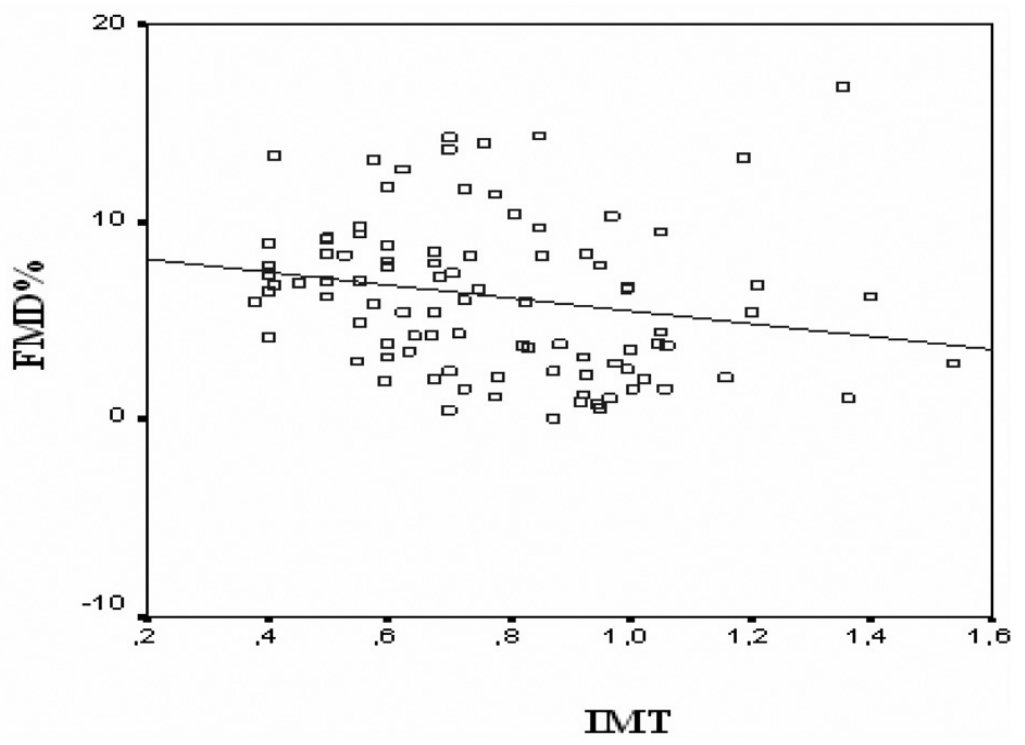

Figure 3. Relationship between flow mediated dilation (FMD) and intima-media thickness (IMT) of all subjects in study group $(r=-0.211, P<0.05)$.

\section{Results}

All CAD patients and control subjects successfully completed the dypridamole perfusion study without major side effects. Individual characteristics, FMD, IMT and CFR for patients with $\mathrm{CAD}$ and for control subjects are reported in Table 1, 2, respectively. Flow mediated dilation was $7.47 \pm$ $2.94 \%$ in control subjects and $5.03 \pm 4.24 \%$ in CAD patients $(\mathrm{P}=0.02)$. Intima-media thickness was $0.64 \pm 0.17$ in control subjects and $0.89 \pm 0.24$ in CAD patients $(\mathrm{P}<0.001)$. Coronary flow reserve was $2.40 \pm 0.80$ in control subjects and $1.75 \pm 0.46$ in CAD patients $(\mathrm{P}<0.001)$. All of the parameters correlated with each other. Coronary flow reserve correlated with carotid IMT $(\mathrm{r}=-0.403, \mathrm{P}<0.001)$ (Fig. 1) and brachial artery FMD $(r=0.232, \mathrm{P}<0.05)$ (Fig. 2). Carotid IMT showed negative correlation with brachial artery FMD $(r=-0.211, P<0.05)$ (Fig. 3).

\section{Discussion}

The results of this study demonstrate that CFR and FMD were lower in CAD patients than control subjects and that CFR impairment correlates with the severity of peripheral endothelial dysfunction.

Increased blood flow is an important cause of endothelium-mediated vasodilation, which is reduced in pathophysiologic conditions [18]. Loss of endothelium-dependent dilatation in systemic arteries occurs in the early preclinical stages of CAD, being associated with known coronary risk factors, such as advanced age, male sex, hypercholesterolemia, hypertension, smoking and diabetes [19]. Endothelial function can be evaluated in coronary and peripheral circulation by monitoring the vasodilatation produced by the administration of endothelium-dependent agonist or by increased blood flow shears [20]. At the myocardial level, CFR is an important indicator of the significance of coronary lesions as well as other pathophysiologic conditions impairing coronary vasomotor function. The brachial circulation and the myocardial circulation differ in terms of microvascular architecture and resistance, pattern of blood flow, metabolic regulation and pathways that are activated to induce hyperemia [21]. Nevertheless, the assumption that endothelial dysfunction detectable in brachial arteries is a manifestation of systemic dysfunction, including the coronary circulation, can not be excluded because of the common underlying pathologic processes involved [22]. In fact, a close correlation between peripheral endothelial function and coronary endothelial function was demonstrated previously in patients with CAD [5]. Previous studies demonstrated that vasomotor dysfunction of epicardial coronary arteries is a predictor of cardiac events in patients without angiographic evidence of CAD [22]. In addition, it was shown that many patients with chest pain and normal vessels at coronary angiography have early atherosclerosis, as documented by intravascular ultrasound, reduced CFR and coronary endothelial dysfunction [23, 24].

\section{The relationship between brachial FMD and transtho- racic CFR}

Transthoracic CFR has been used to evaluate coronary microvascular functions and to evaluate conductive functions of epicardial coronary arteries. Several studies have demonstrated that endothelial function, determined with nonin- 
vasive modalities could predict cardiovascular events [25]. Brachial FMD correlates with measurements of coronary artery endothelial function and predicts future adverse coronary events [5]. However, it does not directly assess coronary circulation but, instead, serves as a surrogate of coronary vascular health. In our study we observed that transthoracic CFR correlates well with brachial FMD. Our results suggest that transthoracic CFR might be used as a surrogate of developing atherosclerosis $(\mathrm{r}=0.232, \mathrm{P}<0.05)$.

\section{The relationship between carotid IMT and CFR}

Takiuchi and others found an association between carotid IMT and transthoracic CFR which is generally regarded as indicative of coronary microcirculatory disturbance [26]. Our results are in agreement with these results with respect to the association between IMT and CFR ( $\mathrm{r}=-0.403, \mathrm{P}<$ 0.001). In published studies, FMD, carotid IMT and brachial artery pulse wave velocity showed stronger predictive power when used together as a surrogate of coronary atherosclerosis $[27,28]$. Consistent with this finding when combined with brachial FMD and carotid IMT, transthoracic CFR might strengthen the combined predictive power of these non-invasive surrogates of atherosclerosis. Therefore, accuracy may be improved by the addition of the predictive power of transthoracic CFR.

\section{The relationship between carotid IMT and brachial FMD}

In patients with atherosclerosis, a significant negative correlation between carotid IMT and brachial FMD has been reported [29]. In a published study Kobayoshi and colleagues suggested that carotid IMT significantly correlates with brachial FMD $(r=-0.343, P<0.001)$. In addition, they suggested that when taken together, FMD, carotid IMT and brachial artery pulse wave velocity have stronger predictive power as a surrogate of coronary atherosclerosis [27]. Our results are in agreement with these results with respect to the association between IMT and FMD $(r=-0.211, \mathrm{P}<0.05)$.

The CFR measurement that reflects coronary microvascular function and endothelial function of epicardial coronary arteries by doppler echocardiography, as a cheaper and easy screening test may be used as a detection method in the assessment of major epicardial coronary arteries [7, 30, 31]. A CFR of $<2$ may be evidence of severe coronary artery disease. In a recent report, Rigo [6] suggested that the use of CFR measurement as a sole diagnostic criterion has structural limitation. The Doppler assessment of coronary flow reserve has some limitations. The assessment of absolute blood velocity can be limited in some patients by the large incident angle between the Doppler beam and blood flow. However, calculation of the flow reserve allows assessment of flow patterns without the need for absolute values. More importantly, the velocity ratio is used as a surrogate of flow reserve. Flow within the coronary artery is not calculated because cross-sectional visualization of the vessel does not allow an accurate measurement of the diameter of the vessel. In addition, discrimination between microvascular and macrovascular disease could not be made by CFR measurement alone. However, CFR can identify mild to moderate stenosis (reduced flow reserve to subischemic levels) and coronary microvascular dysfunction (reduced flow reserve with angiographically normal coronary arteries). Microvascular dysfunction could lead to myocardial ischemia and cardiovascular disease [6]. Therefore measurement of CFR has value to predict future coronary events [32]. CFR may have a very useful role in our daily clinical practice even we think there is need for confirmation of the prognostic role of CFR in larger studies.

\section{Conclusion}

Brachial FMD, Carotid IMT and transthoracic CFR evaluate different aspects of atherosclerosis as well as different sites of the arterial tree. Although each is regarded as being predictive of cardiovascular events, their combination might have more predictive value. However none of these measurements can be used to direct therapy for cardiovascular diseases with the exception of transthoracic CFR, which might be used to direct cardiovascular therapy and to follow the results of therapy. The results of the present study indicate that CFR is significantly lower in patients with CAD than control subjects. In addition, CFR impairment correlates with the severity of peripheral endothelial dysfunction. Follow-up studies are warranted to verify whether reduced CFR identifies a subset of patients who have CAD and who are at increased risk for cardiovascular events. At present, the impossibility of discriminating pathological behaviour of CFR due to microvascular or epicardial stenosis has represented a major limitation in the final diagnosis.

\section{Conflict of Interest}

None declared.

\section{References}

1. Ross R. Atherosclerosis--an inflammatory disease. N Engl J Med. 1999;340(2):115-126.

2. Quyyumi AA. Endothelial function in health and disease: new insights into the genesis of cardiovascular disease. Am J Med. 1998;105(1A):32S-39S.

3. Widlansky ME, Gokce N, Keaney JF, Jr., Vita JA. The clinical implications of endothelial dysfunction. J Am Coll Cardiol. 2003;42(7):1149-1160.

4. Heitzer T, Schlinzig T, Krohn K, Meinertz T, Munzel 
T. Endothelial dysfunction, oxidative stress, and risk of cardiovascular events in patients with coronary artery disease. Circulation. 2001;104(22):2673-2678.

5. Anderson TJ, Uehata A, Gerhard MD, Meredith IT, Knab S, Delagrange D, Lieberman EH, et al. Close relation of endothelial function in the human coronary and peripheral circulations. J Am Coll Cardiol. 1995;26(5):12351241.

6. Rigo F. Coronary flow reserve in stress-echo lab. From pathophysiologic toy to diagnostic tool. Cardiovasc Ultrasound. 2005;3:8.

7. Galderisi M, Capaldo B, Sidiropulos M, D'Errico A, Ferrara L, Turco A, Guarini P, et al. Determinants of reduction of coronary flow reserve in patients with type 2 diabetes mellitus or arterial hypertension without angiographically determined epicardial coronary stenosis. Am J Hypertens. 2007;20(12):1283-1290.

8. Corretti MC, Anderson TJ, Benjamin EJ, Celermajer D, Charbonneau F, Creager MA, Deanfield J, et al. Guidelines for the ultrasound assessment of endothelial-dependent flow-mediated vasodilation of the brachial artery: a report of the International Brachial Artery Reactivity Task Force. J Am Coll Cardiol. 2002;39(2):257-265.

9. Stein JH. Carotid intima-media thickness and vascular age: you are only as old as your arteries look. J Am Soc Echocardiography 2004; 17: 686-89.

10. Cicorella N, Zanolla L, Franceschini L, Cacici G, De Cristan B, Arieti M, Vassanelli C. Usefulness of ultrasonographic markers of carotid atherosclerosis (intimamedia thickness, unstable carotid plaques and severe carotid stenosis) for predicting presence and extent of coronary artery disease. J Cardiovasc Med (Hagerstown). 2009;10(12):906-912.

11. O'Leary DH, Polak JF, Kronmal RA, Manolio TA, Burke GL, Wolfson SK, Jr. Carotid-artery intima and media thickness as a risk factor for myocardial infarction and stroke in older adults. Cardiovascular Health Study Collaborative Research Group. N Engl J Med. 1999;340(1):14-22.

12. Greenland P, Abrams J, Aurigemma GP, Bond MG, Clark LT, Criqui MH . Prevention conference V. Beyond secondary prevention. Identifying the high risk patients for primary prevention. Noninvasive tests of atherosclerotic burden. Writing group III. Circulation 2000; 101: 16-22.

13. Caiati C, Zedda N, Montaldo C, Montisci R, Iliceto S. Contrast-enhanced transthoracic second harmonic echo Doppler with adenosine: a noninvasive, rapid and effective method for coronary flow reserve assessment. J Am Coll Cardiol. 1999;34(1):122-130.

14. Caiati C, Montaldo C, Zedda N, Bina A, Iliceto S. New noninvasive method for coronary flow reserve assessment: contrast-enhanced transthoracic second harmonic echo Doppler. Circulation. 1999;99(6):771-778.
15. Takeuchi M, Lodato JA, Furlong KT, Lang RM, Yoshikawa J. Feasibility of measuring coronary flow velocity and reserve in the left anterior descending coronary artery by transthoracic Doppler echocardiography in a relatively obese American population. Echocardiography. 2005;22(3):225-232.

16. Lambertz H, Tries HP, Stein T, Lethen H. Noninvasive assessment of coronary flow reserve with transthoracic signal-enhanced Doppler echocardiography. J Am Soc Echocardiogr. 1999;12(3):186-195.

17. Korcarz CE, Stein JH. Noninvasive assessment of coronary flow reserve by echocardiography: technical considerations. J Am Soc Echocardiogr. 2004;17(6):704-707.

18. Hermann J, Lerman A. The endothelium: dysfunction and beyond. J Nuc Cardiol 2001; 8: 197-06.

19. Celermajer DS, Sorensen KE, Bull C, Robinson J, Deanfield JE. Endothelium-dependent dilation in the systemic arteries of asymptomatic subjects relates to coronary risk factors and their interaction. J Am Coll Cardiol. 1994;24(6):1468-1474.

20. Vogel RA. Measurement of endothelial function by brachial artery flow-mediated vasodilation. Am J Cardiol. 2001;88(2A):31E-34E.

21. Hirata K, Amudha K, Elina R, Hozumi T, Yoshikawa J, Homma S, Lang CC. Measurement of coronary vasomotor function: getting to the heart of the matter in cardiovascular research. Clin Sci (Lond). 2004;107(5):449460.

22. Taylor AJ, Merz CN, Udelson JE. 34th Bethesda Conference: Executive summary--can atherosclerosis imaging techniques improve the detection of patients at risk for ischemic heart disease? J Am Coll Cardiol. 2003;41(11):1860-1862.

23. Bugiardini R. Normal coronary arteries: clinical implications and further classification. Herz. 2005;30(1):3-7.

24. Basarici I. [Determination of TIMI frame counts and slow coronary flow/ Relationship between the slow coronary flow and carotid artery intima-media thickness]. Anadolu Kardiyol Derg. 2007;7(3):333-334; author reply 334 .

25. Modena MG, Bonetti L, Coppi F, Bursi F, Rossi R. Prognostic role of reversible endothelial dysfunction in hypertensive postmenopausal women. J Am Coll Cardiol. 2002;40(3):505-510.

26. Takiuchi S, Rakugi H, Fujii H, Kamide K, Horio T, Nakatani S, Kawano Y, et al. Carotid intima-media thickness is correlated with impairment of coronary flow reserve in hypertensive patients without coronary artery disease. Hypertens Res. 2003;26(12):945-951.

27. Kobayashi K, Akishita M, Yu W, Hashimoto M, Ohni $\mathrm{M}$, Toba K. Interrelationship between non-invasive measurements of atherosclerosis: flow-mediated dilation of brachial artery, carotid intima-media thickness and pulse wave velocity. Atherosclerosis. 2004;173(1):13-18. 
28. Avsar O, Demir I, Ekiz O, Altekin RE, Yalcinkaya S. [Relationship between the slow coronary flow and carotid artery intima-media thickness]. Anadolu Kardiyol Derg. 2007;7(1):19-23.

29. Hashimoto M, Eto M, Akishita M, Kozaki K, Ako J, Iijima K, Kim S, et al. Correlation between flow-mediated vasodilatation of the brachial artery and intimamedia thickness in the carotid artery in men. Arterioscler Thromb Vasc Biol. 1999;19(11):2795-2800.

30. Kataoka Y, Nakatani S, Tanaka N, Kanzaki H, Yasuda S, Morii I, Kawamura A, et al. Role of transthoracic Doppler-determined coronary flow reserve in patients with chest pain. Circ J. 2007;71(6):891-896.

31. Shimada S, Harada K, Toyono M, Tamura M, Takada G. Using transthoracic Doppler echocardiography to diagnose reduced coronary flow velocity reserve in the posterior descending coronary artery in children with elevated right ventricular pressure. Circ J. 2007;71(12):19121917.

32. Britten MB, Zeiher AM, Schachinger V. Microvascular dysfunction in angiographically normal or mildly diseased coronary arteries predicts adverse cardiovascular long-term outcome. Coron Artery Dis. 2004;15(5):259264. 